International Conference Mathematical and Computational Biology 2011

International Journal of Modern Physics: Conference Series

Vol. 9 (2012) 552-559

(C) World Scientific Publishing Company

DOI: $10.1142 /$ S201019451200565X

\title{
BÉNARD-MARANGONI FERROCONVECTION WITH FEEDBACK CONTROL
}

\author{
NOR FADZILLAH MOHD MOKHTAR* \\ Center of Foundation Studies for Agricultural Science \& Department of Mathematics, \\ Universiti Putra Malaysia, 43400 Serdang, Selangor, Malaysia \\ norfadzillah.mokhtar@gmail.com \\ NORIHAN MD ARIFIN \\ Department of Mathematics \& Institute of Mathematical Research, \\ Universiti Putra Malaysia, 43400 Serdang, Selangor, Malaysia, \\ norihan@math.upm.edu.my
}

\begin{abstract}
The effect of feedback control on the onset of Bénard-Marangoni ferroconvection in a horizontal ferrofluid layer heated from below is investigated theoretically. The lower boundary is rigid and the upper free boundary is assumed to be flat and undeformable. A linear stability analysis is used and the Galerkin method is employed to find the critical stability parameters numerically. It is found that the onset of instability can be delayed through the use of feedback control.
\end{abstract}

Keywords: Ferrofluid; Bénard-Marangoni ferroconvection; feedback control; Galerkin method.

\section{Introduction}

Ferrofluids or known as magnetic fluids are electrically non-conducting colloidal suspensions of tiny particles of solid ferromagnetic material in a non-electrically conducting fluid. An introduction of the ferrofluid is discussed in Ref. 1 and along with that, Ref. 2 review several applications of heat transfer through ferromagnetic fluids. The generalization of the Rayleigh-Bénard problem for magnetic fluids by considering linear stability theory has been studied by many authors. ${ }^{3-8}$

Delaying the onset of convection by the use of linear and nonlinear control was described in Refs. 9-11. The study of the feedback control with a free-slip bottom and the effect of internal heat generation in a fluid layer are discussed in Refs. 12-13. The feedback control with variety of effects in ordinary fluid and micropolar can be seen in Refs. 14-19.

The intent of the present paper is to investigate the coupled Bénard-Marangoni ferroconvection in a ferrofluid layer in the presence of feedback control. The lower 
boundary is assumed to be rigid and upper free boundary is assumed to be flat. The resulting eigenvalue problem is solved by using Galerkin method.

\section{Problem Formulation}

We consider a Boussinesq ferrofluid layer of thickness $d$ with no lateral boundaries and a uniform magnetic field $\vec{H}$ acting normal to the boundaries and parallel to gravity $\vec{g}$. The lower and upper boundaries are maintained at constant temperature with $T_{l}>T_{u}$. A Cartesian co-ordinate system $(x, y, z)$ is used with the origin at the lower boundary and the $z$-axis vertically upward. The lower rigid boundary is assumed to be isothermal to temperature perturbations, while the upper non-deformable free boundary is insulating to temperature perturbations. The governing equations in the Boussinesq approximation are

$$
\begin{gathered}
\nabla \cdot \vec{V}=0, \\
\rho_{0}\left[\frac{\partial \vec{V}}{\partial t}+(\vec{V} \cdot \nabla) \vec{V}\right]=-\nabla p+\rho \vec{g}+\mu_{0}(\vec{Y} \cdot \nabla) \vec{H}+\mu \nabla^{2} \vec{V}, \\
{\left[\rho_{0} C_{V, H}-\mu_{0} \vec{H} \cdot\left(\frac{\partial \vec{Y}}{\partial T}\right)_{V, H}\right] \times \frac{D T}{D t}+\mu_{0} T\left(\frac{\partial \vec{Y}}{\partial T}\right)_{V, H} \cdot \frac{D H}{D t}=k \nabla^{2} T,} \\
\nabla \cdot \vec{B}=0, \\
\nabla \cdot \vec{H}=0, \\
\vec{B}=\mu_{0}(\vec{Y}+\vec{H}), \\
\vec{Y}=\left(Y_{0}+\chi\left[H-H_{0}\right]-J\left(T-T_{0}\right)\right) \frac{\vec{H}}{H},
\end{gathered}
$$

where $\vec{V}=(u, v, w)$ is the velocity vector, $t$ the time, $p$ the pressure, $\vec{H}$ the magnetic field intensity, $\vec{Y}$ the magnetization, $\vec{B}$ the magnetic induction, $\mu$ the dynamic viscosity, $\mu_{0}$ the magnetic permeability of vacuum, $k$ the thermal conductivity, $C_{V, H}$ the specific heat capacity at constant volume and magnetic field per unit mass, $\chi(\partial Y / \partial H)_{H_{0}, T_{0}}$ the magnetic susceptibility, $J=-(\partial Y / \partial T)_{H_{0}, T_{0}}$ the pyromagnetic coefficient and $\nabla$ the Laplacian operator. The basic state is quiescent and is given by

$$
\begin{gathered}
\vec{V}_{b}=0, \\
p=p_{b}(z), \\
T_{b}(z)=T_{0}-\left(\frac{\Delta T}{d}\right) z, \\
\vec{H}_{b}(z)=\left[H_{0}-\frac{J}{1+\chi}\left(\frac{\Delta T}{d}\right) z\right] \hat{k},
\end{gathered}
$$




$$
\vec{Y}_{b}(z)=\left[Y_{0}+\frac{J}{1+\chi}\left(\frac{\Delta T}{d}\right) z\right] \hat{k}
$$

where the subscript $b$ denotes the basic state and $\hat{k}$ is the unit vector in the $z$-direction. To study the stability of the system, the basic state is perturbed in the form

$$
\vec{V}=\vec{V}^{\prime}, \quad p=p_{b}(z)+p^{\prime}, \quad T=T_{b}(z)+T, \quad \vec{H}=\vec{H}_{b}(z)+\vec{H}^{\prime},
$$

where the primed quantities represent the perturbed variables. Substituting Eq. (13) into Eq. (6), using Eqs. (4) - (7), we have

$$
\begin{aligned}
& H_{x}+Y_{x}=\left(1+Y_{0} / H_{0}\right) H_{x}, \\
& H_{y}+Y_{y}=\left(1+Y_{0} / H_{0}\right) H_{y}, \\
& H_{z}+Y_{z}=(1+\chi) H_{z}-J T,
\end{aligned}
$$

where $\left(H_{x}, H_{y}, H_{z}\right)$ and $\left(M_{x}, M_{y}, M_{z}\right)$ are the $(x, y, z)$ components of the magnetic field intensity and magnetization, respectively. Substituting Eq. (13) in Eqs. (2)-(5) and use Eq. (14), eliminating the pressure by operating curl twice, and retaining the $z$ component, we obtain

$$
\begin{gathered}
\left(\rho_{0} \frac{\partial}{\partial t}-\mu \nabla^{2}\right) \nabla^{2} w=-\mu_{0} J\left(-\frac{\Delta T}{d}\right) \times \frac{\partial}{\partial t}\left(\nabla_{h}^{2} \varphi\right)+\rho_{0} \alpha_{t} g \nabla_{h}^{2} T+\frac{\mu_{0} J^{2}}{1+\chi}\left(-\frac{\Delta T}{d}\right) \nabla_{h}^{2} T, \\
\frac{\partial T}{\partial t}-\mu_{0} T_{0} J \frac{\partial}{\partial t}\left(\frac{\partial \varphi}{\partial z}\right)=k \nabla^{2} T+\left[1-\frac{\mu_{0} T_{0} J^{2}}{1+\chi}\right]\left(\frac{\Delta T}{d}\right) w \\
\left(1+\frac{Y_{0}}{H_{0}}\right) \nabla_{h}^{2} \varphi+(1+\chi) \frac{\partial^{2} \varphi}{\partial z^{2}}-J \frac{\partial T}{\partial z}=0 .
\end{gathered}
$$

The normal mode expansion of the dependent variables is assumed in the form

$$
\{w, T, \varphi\}=\{W, \Theta, \Phi\}(z) e^{i(l x+m y)},
$$

where $l$ and $m$ are wave numbers in the $x$ and $y$ directions respectively. Substituting Eq. (18) in Eqs. (15)-(17) and non-dimensionalizing the quantities in the form

$$
\left(x^{*}, y^{*}, z^{*}\right)=\left(\frac{x}{d}, \frac{y}{d}, \frac{z}{d}\right), W^{*}=\frac{d}{v} W, \Theta^{*}=\frac{\kappa \Delta T}{v d^{2}} \Theta, \Phi^{*}=\frac{(1+\chi) \kappa}{J v d \Delta T} \Phi, t^{*}=\frac{v}{d^{2}} t,
$$

where $v=\mu / \rho_{0}$ is the kinematic viscosity and $\kappa$ is the thermal diffusivity, we obtain

$$
\begin{gathered}
\left(D^{2}-a^{2}\right)^{2} W=(R a+R m) a^{2} \Theta-a^{2} R m D \Phi, \\
\left(D^{2}-a^{2}\right) \Theta=-W,
\end{gathered}
$$




$$
\left(D^{2}-J_{3} a^{2}\right) \Phi=D \Theta,
$$

where $D=d / d z$ is the differential operator, $a=\sqrt{l^{2}+m^{2}}$ is the overall horizontal wave number, $J_{1}=\mu_{0} Y^{2} \beta /(1+\chi) \alpha \rho_{0} g$ is the magnetic number, $J_{3}=\left(1+J_{0} / H_{0}\right) /(1+\chi)$ is the measure of nonlinearity of fluid magnetization parameter, $R a=\alpha g \beta d^{4} / \kappa \nu$ is the thermal Rayleigh number and $R m=R a J_{1}$ is the magnetic Rayleigh number.

Following the proportional feedback control in Ref. 11, the continuously distributed actuators and sensors are arranged in a way that for every sensor, there is an actuator positioned directly beneath it. The determination of a control; $q(t)$, can be accomplished using the proportional-integral-differential (PID) controller of the form,

$$
q(t)=r+K[e(t)], \quad e(t)=\hat{m}(t)-m(t),
$$

where $r$ is the calibration of the control, $e(t)$ an error or deviation from the state measurement, $\hat{m}(t)$, from some desired or reference value, $m(t)$, $K=K_{p}+K_{D} d / d t+K_{l} \int_{0}^{t} d t$ with $K_{p}$ is the proportional gain, $K_{D}$ differential gain and $K_{l}$ integral gain. Based on Eq. (23), for one sensor plane and proportional feedback control, the actuator modifies the heated surface temperature using a proportional relationship between the upper $(z=1)$ and lower $(z=0)$ thermal boundaries for perturbation field,

$$
T^{\prime}(x, y, 0, t)=-K T^{\prime}(x, y, 1, t),
$$

where $T$ denotes the deviation of the fluid's temperature from its conductive state. Eqs. (20)-(22) are to be solved subject to appropriate boundary conditions and assumptions.

The boundary conditions considered are

$$
\begin{gathered}
W=D W=\Theta(0)+K \Theta(1)=\Phi=0, \quad \text { at } z=0 \\
W=D^{2} W+a^{2} \Theta M a=D \Theta=D \Phi=0, \quad \text { at } z=1
\end{gathered}
$$

where $M a=\sigma_{T} d \Delta T / \mu \kappa$ is the Marangoni number.

\section{Method of Solution}

Equations (20)-(22) together with the boundary conditions (25) and (26) constitute a problem with the Marangoni number $M a$ or the Rayleigh number $R a$, as an eigenvalue while keeping other physical parameters fixed. To solve the resulting eigenvalue problem, Galerkin's method is used. Accordingly, the variables are written in a series of basis function as

$$
W(z)=\sum_{i=1}^{n} A_{i} W_{i}(z), \quad \Theta(z)=\sum_{i=1}^{n} C_{i} \Theta_{i}(z) \text { and } \Phi(z)=\sum_{i=1}^{n} D_{i} \Phi_{i}(z)
$$

where $A_{i}, C_{i}$ and $D_{i}$ is the constants to be determined. The trial functions $W_{i}(z), \Theta_{i}(z)$ and $\Phi_{i}(z)$ will be generally chosen in such a way that they satisfy the respective boundary conditions. We select the trial functions as 


$$
W_{i}=z^{4}-\frac{5 z^{3}}{2}+\frac{3 z^{2}}{2}, \quad \Theta_{i}=z^{2}-2 z \text { and } \Phi_{i}=z\left(z^{2}-\frac{11 z}{6}+\frac{2}{3}\right) .
$$

Substituting Eq. (27) into Eqs. (20)-(22), multiplying the resulting momentum Eq. (20) by $W_{j}(z)$, energy Eq. (21) by $\Theta_{j}(z)$ and magnetic potential Eq. (22) by $\Phi_{j}(z)$; performing the integration by parts with respect to $z=0$ and 1 , and using the boundary conditions (25) and (26), we obtain an expression for $M a$, after using Eq. (28) in the form

$$
M a=\frac{\alpha_{1}}{1080 a^{2}}\left(8 a^{2}+20+15 K\right)-\frac{4}{35}(R a+R m)+\frac{61}{\alpha_{2}} R m,
$$

where

$$
\begin{aligned}
& \alpha_{1}=19 a^{4}+432 a^{2}+4536 \\
& \alpha_{2}=20\left(434+29 a^{2} J_{3}\right) .
\end{aligned}
$$

\section{Results and Discussion}

The linear stability analysis is carried out to investigate the effect of feedback control on the onset of coupled Bénard-Marangoni ferroconvection in a horizontal ferrofluid layer. In order to validate the numerical solution procedure used, our results are compared with the previously published results of Ref. 8 as shown in Table 1 . The results tabulated in Table 1 for different values of Rayleigh number $R a$ and controller $K$ are for $R m=0$ and $J_{3}=0$. From the table it is evident that there is an excellent agreement between the results of the present study and the previously published ones. This verifies the applicability and accuracy of the method used in solving the problem. As for the effect of the controller $K$ in the system, we found that an increase of the $K$, increased the value of the critical Marangoni number; $M a_{c}$, for all Rayleigh number; $R a$, considered. However, an increase of the $R a$, decrease the value of the $M a_{c}$ and the system become unstable for all $K$ stated.

Table 1. Comparison of $M a_{c}$ for different values of $R a$ and $K$ with $R m=0$.

\begin{tabular}{cccccc}
\hline \multirow{2}{*}{$R a$} & \multicolumn{5}{c}{$M a_{c}$} \\
\cline { 2 - 6 } & Ref. 8 & \multicolumn{5}{c}{ Present Analysis } \\
\cline { 2 - 6 } & $K=0$ & $K=0$ & $K=1$ & $K=3$ & $K=5$ \\
\hline 0 & 79.00 & 79.00 & 99.51 & 135.76 & 169.25 \\
100 & 67.57 & 67.57 & 88.08 & 124.33 & 157.82 \\
200 & 56.14 & 56.14 & 76.65 & 112.90 & 146.39 \\
300 & 44.71 & 44.71 & 65.23 & 101.47 & 134.96 \\
400 & 33.29 & 33.29 & 53.80 & 90.04 & 123.53 \\
500 & 21.36 & 21.86 & 42.37 & 78.62 & 112.11 \\
600 & 10.43 & 10.43 & 30.94 & 67.19 & 100.68 \\
\hline
\end{tabular}


Figure 1 and Fig. 2 show the variation of Marangoni number; $M a$, and Rayleigh number; $R a$, respectively with wave number; $a$, for different values of $K$ when $J_{3}=1$. From the graphs it clearly show that, an increase of the controller $K$, increase the value of the Marangoni number and Rayleigh number as well. The marginal stability curves shift upwards for both cases considered and this is an evident that the controller stabilize the no-motion state for all wave numbers; $a$.

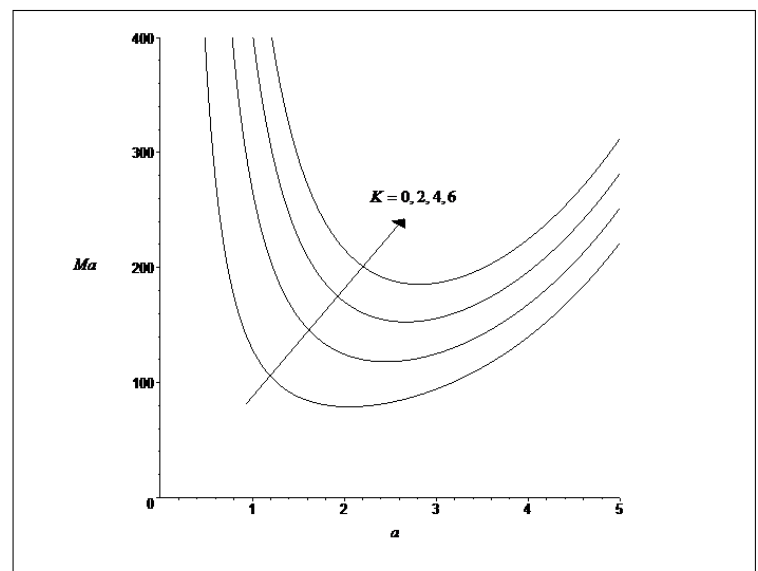

Fig. 1. Variation of $M a$ with $a$ for different values of $K$ when $R a=0$ and $R m=0$.

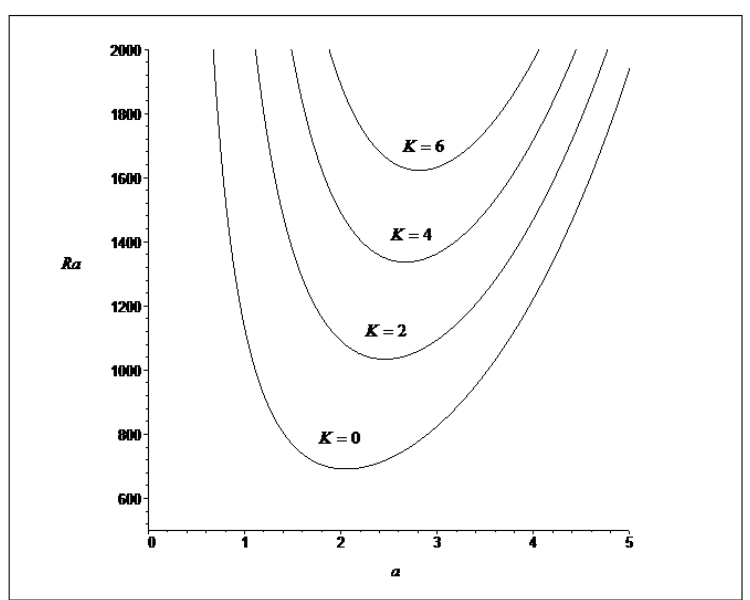

Fig. 2. Variation of $R a$ with $a$ for different values of $K$ when $M a=0$. 
Figure 3 and Fig. 4 show the variation of $M a_{c}$ and $R a_{c}$ respectively, with controller $K$ for different values of $R m$ when $J_{3}=1$. We choose $R m=0,10$ and 20 for both cases in order to investigate the effect of the magnetic Rayleigh number in the presence of controller $K$. We observed that an increase of $R m$, decrease the value of $M a_{c}$ and $R a_{c}$ which in turns destabilize the system. This is due to additive reinforcement of destabilizing magnetic force. From the graphs, it is also evident that the controller $K$ is a stabilizing factor for the system to become more stable.

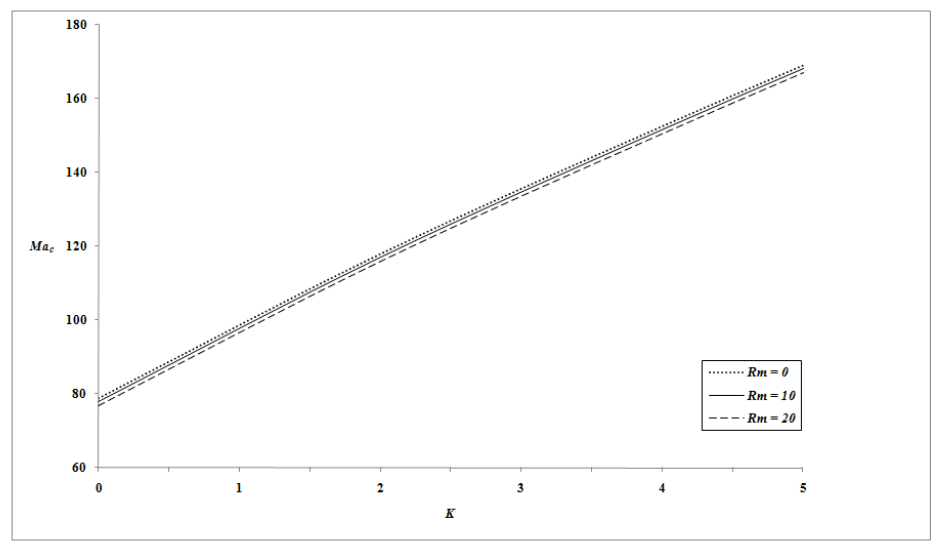

Fig. 3. Variation of $M a_{c}$ with $K$ for different values of $R m$ when $R a=0$.

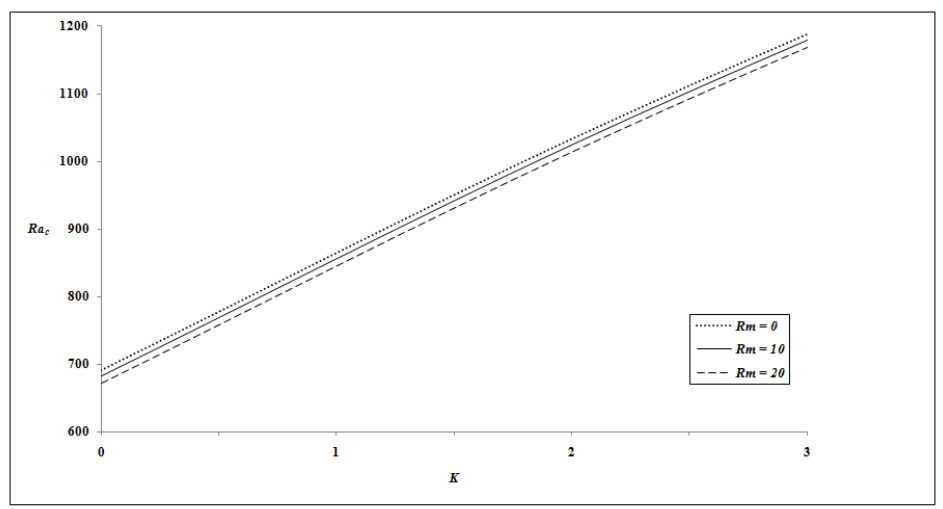

Fig. 4. Variation of $R a_{c}$ with $K$ for different values of $R m$ when $M a=0$. 


\section{Conclusions}

The effect of feedback control on the onset of coupled Bènard-Marangoni ferroconvection is studied theoretically. From the foregoing study, we found that the effect of increasing the feedback control in the ferrofluid system is to elevate the critical Marangoni number and the critical Rayleigh number, thus promote stability in the coupled Bénard-Marangoni ferroconvection. However, the increase in the value of magnetic Rayleigh number $R m$, is to reinforce and hasten the onset of coupled BénardMarangoni ferroconvection and the system become more unstable.

\section{Acknowledgements}

The authors acknowledge the financial support received from Ministry of Higher Education Malaysia under the FRGS scheme.

\section{References}

1. R. E. Rosensweig, Ferrohydrodynamics, (Cambridge University Press, Cambridge, 1985).

2. V. G. Bashtvoy, B. M. Berkovsky and A. N. Vislovich, Introduction to Thermomechanics of Magnetic Fluids, (Hemisphere Co., Washington D.C, 1988).

3. B. A. Finlayson, J. Fluid Mech. 40, 753 (1970).

4. M. Das Gupta and A. S. Gupta, Int. J. Eng. Sci. 17, 271 (1979).

5. K. Gotoh and M. Yamada, J. Phys. Soc. Japan. 51, 3042 (1982).

6. P. J. Stiles and M. J. Kagan, J. Colloid Interface Sci. 132, 435 (1990).

7. S. Venkatasubramanian and P. N. Kaloni, Int. J. Eng. Sci. 32, 237 (1994).

8. I. S. Shivakumara, N. Rudraiah and C. E. Nanjundappa, J. Magnetism \& Magnetic Mat. 248, 379 (2001).

9. J. Tang and H. H. Bau, J. Fluid Mech. 257, 485 (1993).

10. J. Tang and H. H. Bau, Phys. Fluids. 10, 1597 (1998).

11. H. H. Bau, J. Heat Mass Trans. 42, 1327 (1999).

12. N. M. Arifin, R. Nazar and N. Senu, J. Phys. Society Japan. 76, 14401 (2007).

13. N. Bachok and N. M. Arifin, Microgravity Sci. Technol. 22, 97 (2010).

14. I. Hashim and Z. Siri, Numeric. Heat Transfer A: Appl. 54, 1368 (2008).

15. Z. Siri and I. Hashim, Int. Commun. Heat Mass Transfer. 35, 1130 (2008).

16. Z. Siri and I. Hashim, Sains Malaysiana. 38, 119 (2008).

17. S. A. Kechil and I. Hashim, Int. Commun. Heat Mass Transfer. 35, 647 (2008).

18. Z. Siri, Z. Mustafa and I. Hashim, Int. J. Heat Mass Transfer. 52, 5770 (2009).

19. M. N. Mahmud, Z. Mustafa and I. Hashim, Int. Commun. Heat Mass Transfer. 37, 1335 (2010). 